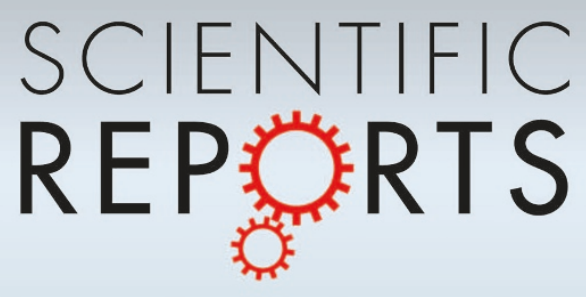

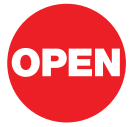

SUBJECT AREAS: PLURIPOTENCY

EMBRYONIC STEM CELLS

CANCER MODELS

MECHANISMS OF DISEASE

Received

17 July 2012

Accepted

29 October 2012

Published

10 December 2012

Correspondence and requests for materials should be addressed to T.O. (tochiya@ncc.go.

ip)

\section{Two distinct knockout approaches highlight a critical role for p53 in rat development}

\author{
Masaki Kawamata \& Takahiro Ochiya
}

Division of Molecular and Cellular Medicine, National Cancer Center Research Institute, 1-1, Tsukiii, 5-chome, Chuo-ku, Tokyo $104-$ 0045, Japan.

Gene targeting in embryonic stem cells (ESCs) has become the principal technology for generating knockout models. Although numerous studies have predicted that the disruption of $p 53$ leads to increased developmental anomalies and malignancies, most p53 knockout mice develop normally. Therefore, the role of p53 in animal development was examined using rat knockout models. Conventionally generated homozygous KO males developed normally, whereas females rarely survived due to neural tube defects. Mutant chimeras generated via blastocyst injection with p53-null ESCs exhibited high rates of embryonic lethality in both sexes. This phenotype could be observed in one month by the use of zinc-finger nucleases. The p53-null ESCs were resistant to apoptosis and differentiation, and exhibited severe chromosome instabilities in the chimera-contributed cells, suggesting an essential role for p53 in maintaining ESC quality and genomic integrity. These results demonstrate that p53 functions as a guardian of embryogenesis in the rats.

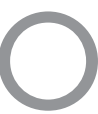
ver the past two decades, knockout (KO) technology in mice has helped to clarify the physiological function of a large number of genes. However, unexpected phenotypes have been observed in some cases, making it difficult to understand the role of the deleted gene, or to translate that data to the phenotypes of human diseases caused by mutations in such genes. Thus, gene-targeting techniques for other animals, such as rats, have long been sought. Many strategies for manipulating rat genes to generate loss-of-function models have been adapted from the mouse genetic toolbox, including conventional transgenesis by pronuclear injection ${ }^{1}$, RNA interference ${ }^{2}$, N-ethyl-N-nitrosourea (ENU) mutagenesis ${ }^{3,4}$, and transposon mutagenesis ${ }^{5-7}$. KO rats have been produced using Zinc-finger nuclease (ZFN) technology ${ }^{8,9}$, and, most recently, germline-competent rat ESCs and rat induced pluripotent stem cells (iPSCs) have been established by the addition of cell-signaling inhibitors to the culture medium ${ }^{10-13}$, making it possible to generate both transgenic $(\mathrm{Tg})^{12,14}$ and $\mathrm{KO}$ rats $^{15}$.

The tumor suppressor gene $p 53$ is a good example of a gene whose function in mouse development requires further scrutiny. Donehower et al. first reported normal Mendelian ratios and postnatal development in p53 homozygous $\mathrm{KO}$ mice $^{16}$. However, two other groups later showed that a fraction of homozygous KO females had fatal embryonic exencephaly, a defect in neural tube closure that results in an overgrowth of neural tissue in the midbrain region ${ }^{17,18}$. Such results indicate that, at least in some cases, p53 influences development in females ${ }^{19}$. In the case of p53 homozygous KO rats, neural tube defects (NTDs) in females were not found but increased susceptibility to tumor development was reported ${ }^{15,20,21}$.

p53 has been shown to regulate not only cell cycle arrest, apoptosis, and DNA repair in many types of cells ${ }^{22}$, but also stemness, by suppressing Nanog expression in $\mathrm{ESCs}^{23}$. Considering these observations, malignant transformations may occur in p53-null ESCs and chimera development may be hindered. However, chimeric mutant mice have been successfully generated via the injection of blastocysts with iPSCs derived from $p 53$-null mouse embryonic fibroblasts (MEFs), and germline transmission of the $p 53$-null cells was also accomplished ${ }^{24,25}$. The properties of rat ESCs differ from those of mouse ESCs in that rat ESCs cannot be cultured in mouse ESC culture conditions due to their high sensitivity to differentiation signals ${ }^{26}$. Thus, an approach using $p 53$-null rat ESCs might reveal new insights into the function of p53 in regulating stemness and animal development.

We previously generated Oct4-Venus Tg rats, and established Oct4-Venus ESC lines in which Oct4 expression can be monitored by green fluorescence ${ }^{12,27}$. Here, both conventionally $\mathrm{KO}$ and mutant chimeras rats were generated using p53-null Oct4-Venus ESCs, and their development was investigated. Moreover, an efficient 
method for a rapid generation of mutant chimeras was developed using ZFN-mediated gene targeting in rat ESCs. Using this method, developmental phenotypes can be observed within 1 month.

\section{Results}

Conventionally generated $p 53^{-1-}$ females reveal the cause of NTDs. p53 homozygous $\mathrm{KO}$ rats were generated via germline transmission of heterozygous $p 53^{+/ C}$ ESCs (Fig. 1a,b,e). The details are described in the Materials and Methods. The Mendelian ratios in weaned rats produced from heterozygous intercrosses were investigated (Fig. 2a). The frequency of homozygous $p 53^{\mathrm{C} / \mathrm{C}}$ rats was $16.9 \%$, less than the anticipated value of $25 \%$ (Table 1 ). Moreover, only one $p 53^{\mathrm{C} / \mathrm{C}}$ female developed normally, frequency $=0.70 \%$, significantly less than $16.2 \% p 53^{C / C}$ males. These results suggest that most of the $p 53^{C / C}$ females either do not survive gestation, or die after birth but prior to weaning. To investigate the developmental dysfunctions in $p 53^{C / C}$ females, litters from heterozygous intercrosses were examined at embryonic day 16.0 (E16.0) to E18.0. Eleven $p 53^{C / C}$ female embryos $(12.8 \%, 11 / 86)$ were recovered at this stage; six $(57 \%, 6 /$ 11) exhibited exencephaly (Table 1) and two of these also exhibited spina bifida (Fig. 2b). Although these two abnormalities are the most prevalent NTDs, spina bifida in p53 mutant mice has only been reported in one study ${ }^{28}$. Exencephaly was only found in the female embryos, consistent with previous observations of a higher incidence of NTDs in human females and in numerous mouse models ${ }^{29}$. Expression of SOX2, a marker for primordial neuronal cells

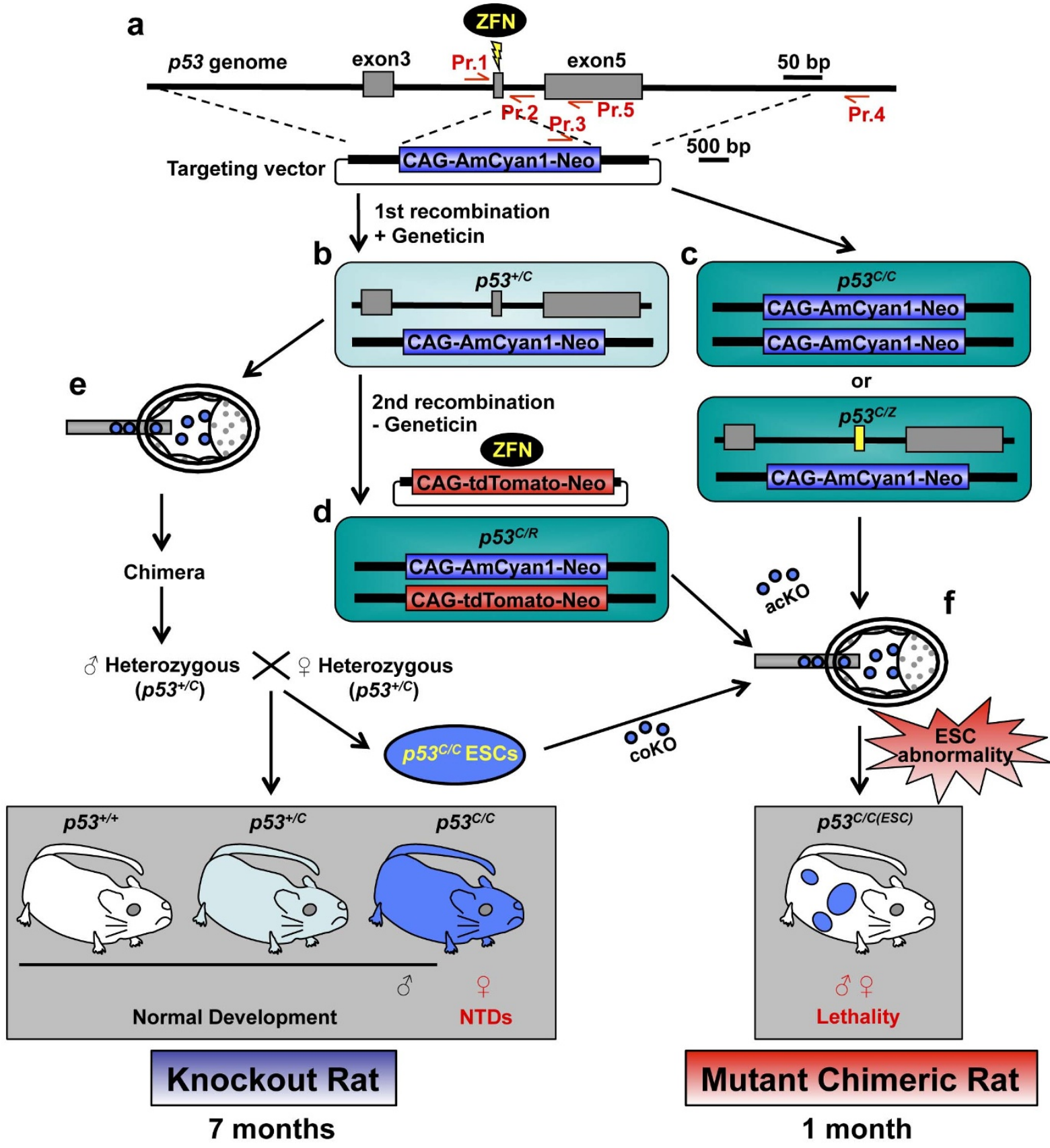

Figure 1 Schematic representation of p53 KO strategy in rats. (a-d) Both mono- (b) and bi-allelic (c) or 2ndary (d) homologous recombination are induced by ZFN. (e, f) Heterozygous or homozygous ESC-injection leads to the generation of conventionally generated KO model (e) or ESC-based mutant chimeric models (f), respectively. A yellow box indicates a frame shift mutation induced by ZFNs. Pr., Primer. coKO, congenital KO. acKO, acquired $\mathrm{KO}$. 
Table 1 | The p53 Genotypes of Adult and Embryonic Rats

\begin{tabular}{lcccc} 
Genotype & $\mathrm{p} 53^{+/+}$ & $\mathrm{p} 53^{+/ c}$ & $\mathrm{p} 53^{\mathrm{c} / \mathrm{c}}$ & $\begin{array}{c}\mathrm{p} 53^{\mathrm{c} / \mathrm{c}} \\
\text { Exencephaly }\end{array}$ \\
\hline Adults & $41(28.9 \%)$ & $77(54.2 \%)$ & $24(16.9 \%)$ & 0 \\
Male & $18(12.7 \%)$ & $42(29.6 \%)$ & $23(16.2 \%)$ & 0 \\
Female & $23(16.2 \%)$ & $35(24.6 \%)$ & $1(0.70 \%)$ & 0 \\
Embryos & $21(24.4 \%)$ & $48(55.8 \%)$ & $17(19.8 \%)$ & 6 \\
Male & $11(12.8 \%)$ & $22(25.6 \%)$ & $6(7.0 \%)$ & 0 \\
Female & $10(11.6 \%)$ & $26(30.2 \%)$ & $11(12.8 \%)$ & $6(54.5 \%)^{a}$ \\
\hline${ }^{\circ}$ Of the $11 ~ p 53^{c / C}$ female embryos, six exhibited exencephaly. \\
\hline
\end{tabular}

expressed in the embryonic neural plate $\mathrm{e}^{30}$, was detected on the surface of the brain and in areas of spina bifida (Fig. 2d, arrowheads), confirming that neural tube closure had failed. Compared to a $p 53^{+/ C}$ embryo (Fig. 2e, right), the aberrant ventricular zone (VZ) structure in the brain of a $p 53^{\mathrm{C} / \mathrm{C}}$ exencephalic embryo was revealed by the localization of SOX2 (Fig. 2d left, arrows), which is expressed in the neuroendothelial stem cells of the $\mathrm{VZ}^{31}$. In this embryo, Oct4-Venus expressing cells were aberrantly located in the exencephalic region (Fig. $2 b$, green square, and $2 c$ ).

Embryonic lethality in a mutant chimeric model. ZFNs can create site-specific double-strand breaks, which are repaired via non- homologous end joining, resulting in frame-shift mutations by the arbitrary addition or deletion of base pairs. Cotransfection of ZFNs with targeting vectors enhances homologous recombination, not only in human pluripotent cells ${ }^{32,33}$, but also in one-cell embryos, leading to the direct generation of knock-in mice ${ }^{34}$ and rats ${ }^{35}$. In the present work, ZFNs were used to produce homozygous mutant ESC lines by a single recombination step (Fig. 1c). Using this approach, 1 of $46(2.2 \%)$ clones harbored dual knock-in alleles $\left(p 53^{C / C}\right)$, while 7 of $46(15 \%)$ clones possessed both knock-in and frame shift mutant alleles $\left(p 53^{\mathrm{Cl} /}\right)$. In a 2 nd-step recombination, homozygous clones were also produced from a $p 53^{+/ C 3}$ ESC clone based on the same strategy using both ZFNs and a targeting vector expressing red fluorescence (Fig. 1b,d). A successful homologous recombination was achieved in 3 of 8 clones $\left(38 \%, p 53^{C / R}\right.$; Supplementary Fig. S2c). These ESC lines were called acquired KO (acKO) ESCs (Fig. 1c). The contribution of $p 53^{-/-}\left(p 53^{C / C}, p 53^{C / Z}\right.$, or $\left.p 53^{C / R}\right)$ ESCs to rats, which are called $p 53^{-/-(E S C)}$ rats, was examined, and the timeline for the rapid generation of the mutant chimeras is shown schematically (Fig. 3a,b). Microinjection of $p 53^{-/-}$ESCs into blastocysts led to the delivery of only a few pups $(0.4 \pm 0.2$ per foster mother, $n=5,4$ cell lines). This number $(0.4 \pm 0.2 /$ foster mother $)$ was significantly smaller than the number of pups delivered following injection of $p 53^{+/+}$ESCs into blastocysts $(4.0 \pm 1.1$ per foster mother, $\mathrm{n}=5,3$ cell lines, $P<0.05)$ or $p 53^{+/-}\left(p 53^{+/ C}\right)$ ESCs $(5.1$ \pm 1.1 per foster mother, $n=9,4$ cell lines, $P<0.05$ ) (Fig. $3 c$ ). The a

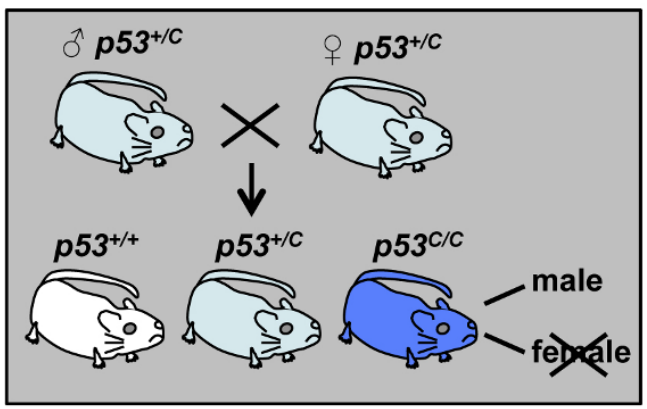

b

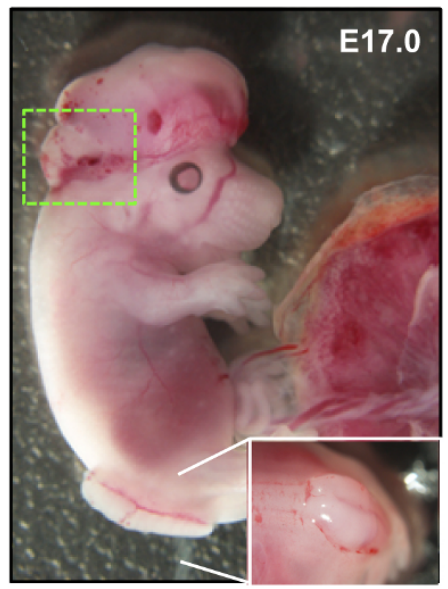

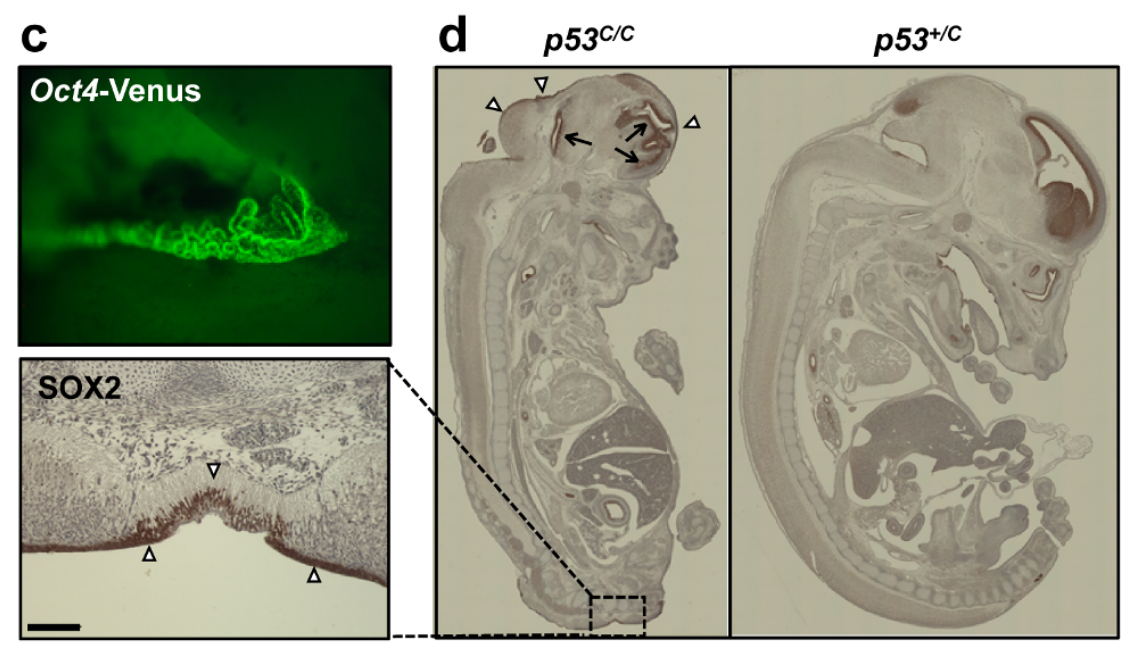

Figure $2 \mid$ Phenotypes in conventionally generated p53 homozygous rats. (a) Schematic representation of heterozygous intercrosses indicates a loss of adult female. (b) An embryo at day 17.0 of gestation (E17.0) displaying exencephaly and spina bifida. A dotted green square indicates (c). (c) Fluorescence image of the area inside the dotted green square in (b). Oct4-Venus fluorescence is observed in the exencephalic region. (d) IHC staining for SOX2 identifies positive cells in the ventricular zone (arrows) and surface (arrowheads) of both brain and spina bifida (magnified image, scale bar $=100 \mu \mathrm{m})$. 
a

\begin{tabular}{|c|c|c|c|}
\hline \multirow[t]{2}{*}{ Targeting } & Cloning & Injection & Phenotype \\
\hline & ESC culture & \multicolumn{2}{|c|}{ Embryo developme } \\
\hline Day: 0 & 9 & 20 & $\begin{array}{cc}31 & 34 \\
(E 14) & (E 17)\end{array}$ \\
\hline
\end{tabular}

b

C

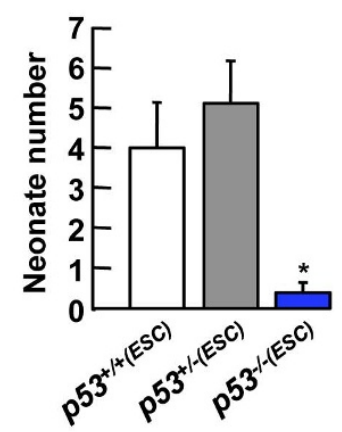

$f$

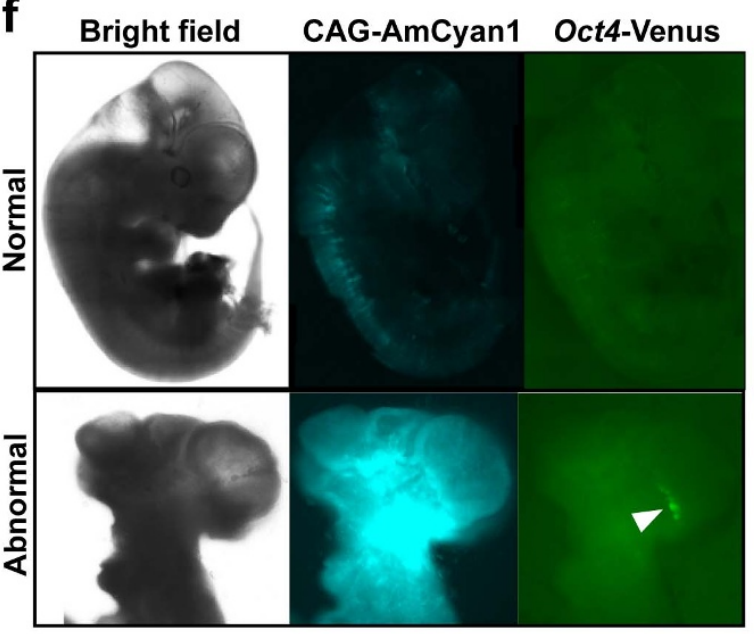

d

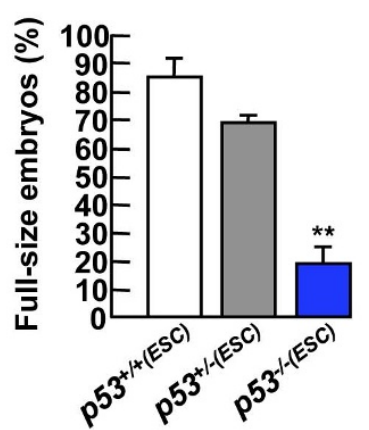

e

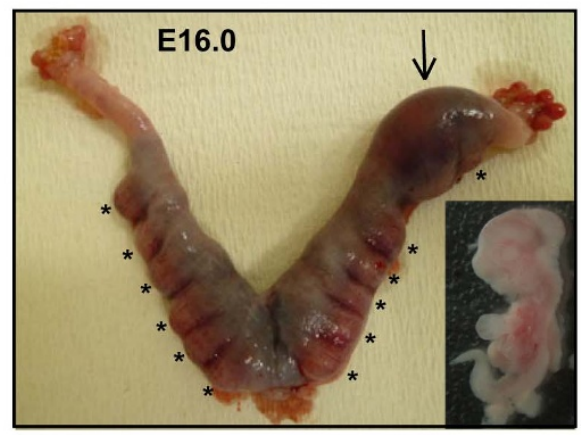

g

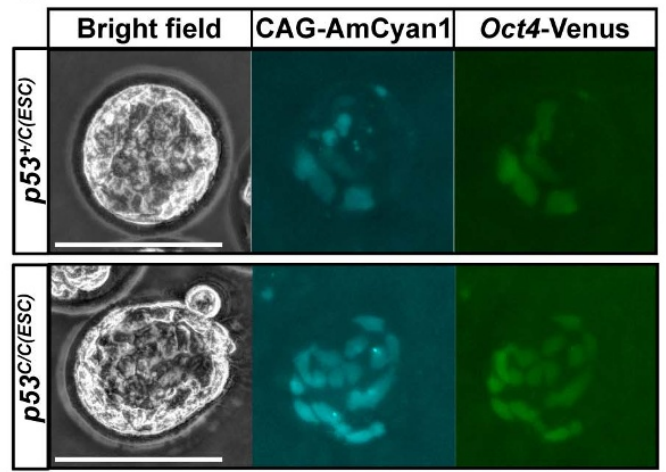

i

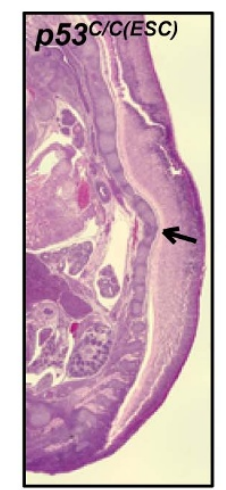

Figure 3 Embryonic lethality in $\mathbf{p} 53$ mutant chimeras. (a) Time line for generating $p 53^{-/-(E S C)}$ chimeras and (b) Schematic representation to investigate phenotype during embryogenesis. (c) Number of neonates successfully delivered. $p 53^{+/+}\left(\mathrm{n}=5,3\right.$ cell lines), $p 53^{+/-}\left(\mathrm{n}=9,4\right.$ cell lines) or $p 53^{-/-}$( $\mathrm{n}=5,4$ cell lines) ESCs were injected into wild-type blastocysts. n, injection number. ${ }^{*}, P<0.05, p 53^{-/-}$vs. $p 53^{+/+}$and $p 53^{+/-}$. (d) The ratio of chimeric embryos with normal body size at E14.0 to E17.0. $p 53^{+/+}\left(\mathrm{n}=4,4\right.$ cell lines), $p 53^{+/-}(\mathrm{n}=7,4$ cell lines $)$ or $p 53^{-/-}(\mathrm{n}=14$, five cell lines $)$ ESCs were injected into wildtype blastocysts. $\mathrm{n}$, injection number. ${ }^{* *}, P<0.001, p 53^{-/-}$vs. $p 53^{+/+}$and $p 53^{+/-}$. (e) Developmental dysfunction in chimeric embryos injected with $p 53^{C / R 2}$ ESCs at E16.0. An arrow indicates a chimera with growth retardation (inset). Asterisks indicate fetal absorption. (f) Correlation between developmental dysfunction and ESC contribution. $p 53^{C / C I}$ ESCs expressing AmCyan 1 contribute to chimeric embryos at E14.0. An arrowhead indicates an ectopic expression of Oct4-Venus. (g) $p 53^{C / R 4}$ ESC proliferation in blastocyst. Twelve ESCs were injected into blastocyst, followed by incubation overnight in YPAC medium. (h) Immunohistochemistry using Cleaved-Caspase3 antibody in liver of $p 53^{+/ C(E S C)}$ or $p 53^{C / C(E S C)}$ chimera. Arrowheads indicate the apoptotic cells. (i) Spinal curvature (an arrow) in $p 53^{C / C(E S C)}$ chimera. All scale bars $=100 \mu \mathrm{m}$.

newborns derived from $\mathrm{p} 53^{-1-}$ ESC-injections did not exhibit a brown coat-color, indicating that they were not chimeras. Because these results suggest that the development of $p 53^{-/-(E S C)}$ embryos was defective, fetal development at stages E14.0 to E17.0 was examined. Approximately $80 \%$ of the $p 53^{-/-(E S C)}$ embryos $(n=14,5$ cell lines, Fig. 3d) showed abnormal development resulting in complete 
resorption (Fig. 3e, asterisks) or growth retardation (Fig. 3e, an arrow and inset). A large number of $p 53^{-/-}$ESC-derived cells were detected in these embryos (Fig. 3f, lower). However, the remaining $p 53^{-1-(E S C)}$ embryos (30/189 embryos: $20.0 \pm 5.6 \%)$ developed a normal body size (Fig. 3f, upper and Supplementary Table S1). The number of normal embryos $(20.0 \pm 5.6 \%)$ was significantly lower than that of $p 53^{+/+(E S C)}$ embryos (53/63 embryos: $85.1 \pm 5.8 \%, \mathrm{n}=4,4$ cell lines, $P<0.01$ ) or $p 53^{+/-(E S C)}$ embryos (44/64 embryos: $69.0 \pm 2.2 \%, \mathrm{n}=7,4$ cell lines, $P<0.01)$ (Fig. 3d). Among the normal-sized $p 53^{-1-(E S C)}$ embryos, 26 of 30 ( $87.8 \pm 9.7 \%, n=10,5$ cell lines) embryos were chimeras, whereas 22 of 26 displayed a relatively lower contribution of the mutant cells. Although the number of $p 53^{+/-(E S C)}$ chimera (30/ 44 embryos, $66.9 \pm 6.6 \%, \mathrm{n}=7,4$ cell lines) was similar to that of $p 53^{-1-(E S C)}$ chimera $(P=0.14)$, the $p 53^{+-(E S C)}$ chimeras developed normally (Fig. $3 c, d)$. The number of $p 53^{+/+(E S C)}$ chimera $(17 / 53$ embryos, $36.0 \pm 10.2 \%, \mathrm{n}=4,4$ cell lines) was significantly smaller than that of either $p 53^{+/-(E S C)}(P=0.044)$ or $p 53^{-/-(E S C)}(P=0.0081)$ chimera. These results suggest that $p 53$ mutation enhances the chimeric contribution of ESCs and the high contribution of $p 53$ null ESCs induces embryonic lethality.

To address the mechanisms by which $p 53^{-/-}$ESCs result in embryonic lethality, the behavior of $p 53^{-/-}$ESCs was followed in blastocysts incubated in vitro. Blastocysts were injected with 12 ESCs and incubated over night. Although $p 53^{+/+1}$ ESCs remained
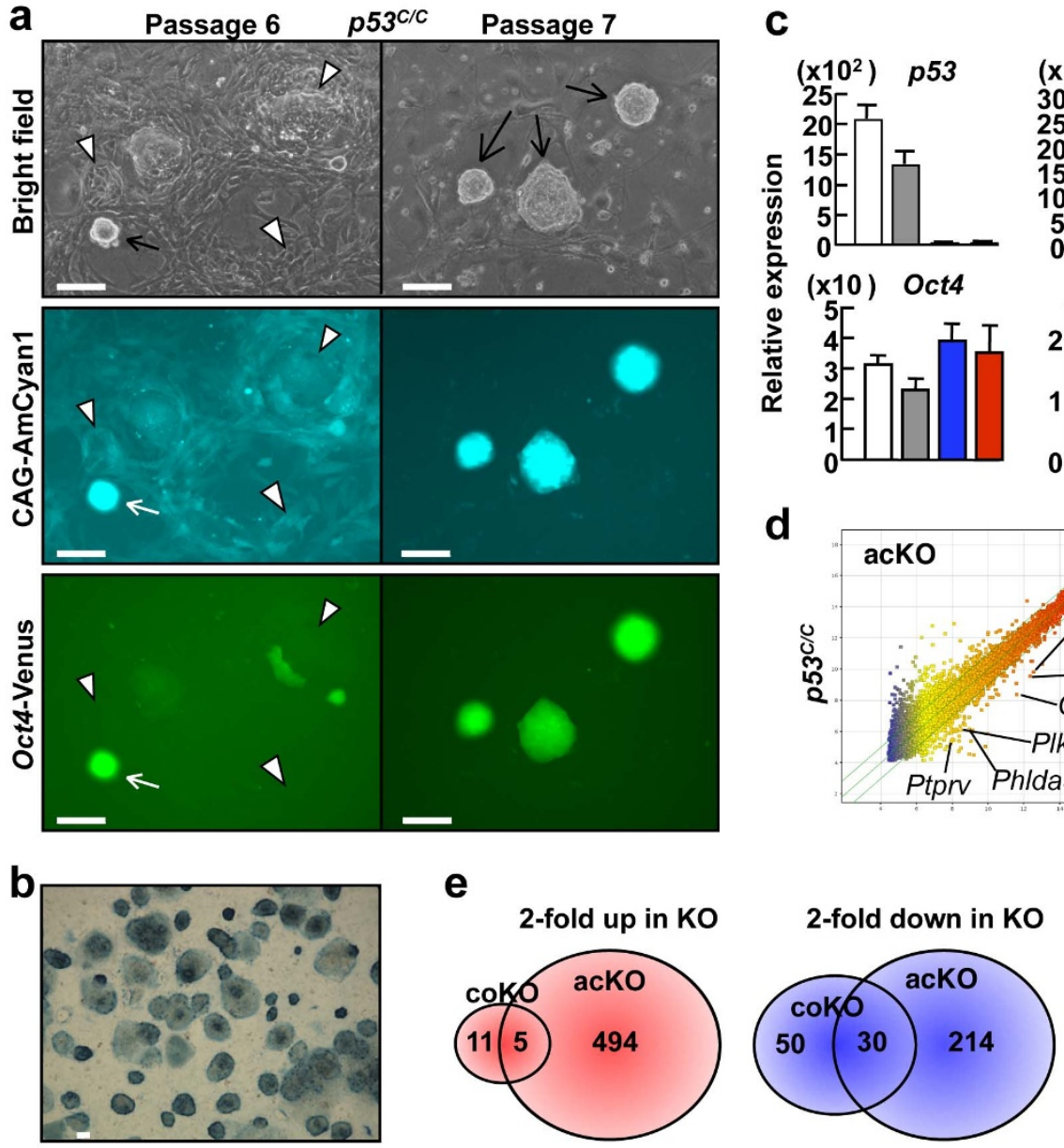

in the blastocysts, the number decreased to $4.0 \pm 0.89$ cells (Fig. $3 g$, upper, $\mathrm{n}=5)$. In contrast, a significantly larger number of $p 53^{\mathrm{C} / R 4}$ ESCs (13.7 \pm 1.2 cells, $n=7, P<0.0001$ ) were detected (Fig. 3g, lower), indicating proliferation of the p53-null ESCs in the blastocysts. The excess proliferation may lead to a high ESC contribution, resulting in the developmental abnormalities that led to resorption of the fetuses. In fact, several of the $p 53^{C / C(E S C)}$ chimeras with normal body size displayed increased number of apoptotic cells in the liver (Fig. 3h, arrowheads) and one chimera exhibited an abnormal spinal curvature (Fig. 3i, an arrow). Embryos such as these may die and undergo resorption before birth, resulting in the significant loss of neonates as shown in Figure $3 \mathrm{~d}$.

Morphology and global gene expression profile in $p 53^{-/-}$ESCs. The properties of $p 53^{-/-}$ESCs were examined. Venus-negative differentiated cells (Fig. 4a, left, arrowheads) survived the processes of cloning and passaging $p 53^{-/-}$Venus-positive ESCs (Fig. 4a, left, arrows), indicating that $p 53^{-/-}$differentiated cells escaped from apoptosis. Rat ESC colonies adopt a dome-shaped morphology and tend to detach from culture dishes coated with MEFs ${ }^{12,15}$. The $p 53^{-1}$ domed colonies were detached by pipetting and the cells were passaged after dissociation, leading to successful propagation of dome-shaped colonies; no differentiated cells were detected. (Fig. 4a, right, arrows). The morphology of the $p 53^{-1-}$ lines was

C
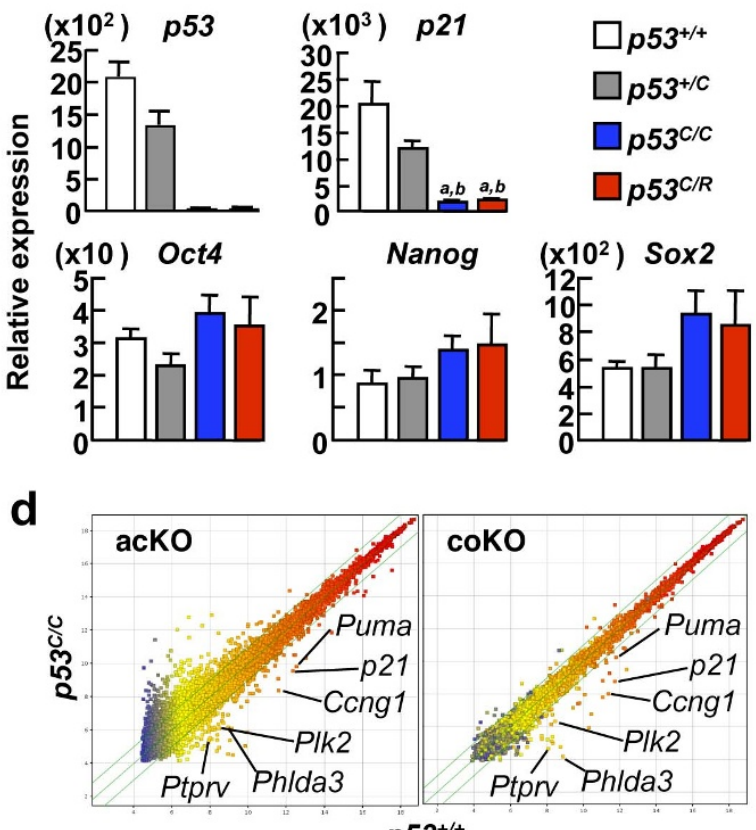

$\mathrm{p53}^{+/+}$

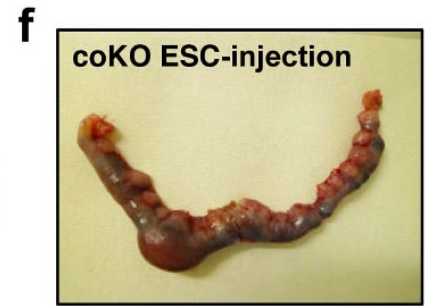

Figure $4 \mid$ Characteristics of $p 53$-null ESCs. (a) A $p 53^{C / C 1}$ ESC clone is shown. Arrows indicate pluripotent colonies. Arrowheads indicate differentiated cells. (b) ALP staining in $p 53^{C / C l}$ ESCs. (c) q-PCR analysis in $p 53$ mutant ESCs. Transcript levels were normalized to Gapdh levels. Data are the mean \pm SD of one biological sample assayed in four independent experiments. $a, P<0.05$ versus $p 53^{+/+} ; b, P<0.005$ versus $p 53^{+/ C}$. (d) Scatter plots of global gene expression microarrays comparing $p 53^{+/+}$and $p 53^{C / C}$ ESCs of an acKO (left) or coKO (right) line. The green lines delineate the boundaries of a 2 -fold difference in gene expression levels. (e) Venn diagrams of the intersection between genes highly (left) or lowly (right) expressed in the coKO versus the acKO in $p 53^{C / C}$ ESCs. (f) Developmental dysfunction in chimeric embryos injected with $p 53^{C / C 2}$ coKO ESCs at E16.0. All scale bars $=100 \mu \mathrm{m}$. 
indistinguishable from that of $p 53^{+/-}$or $p 53^{+/+}$cells (Supplementary Fig. S3). The $p 53^{C / C}$ ESCs were positive for alkaline phosphatase (ALP) activity (Fig. 4b).

The expression levels of ESC marker genes, such as Oct4, Nanog and Sox2, were similar in $p 53^{C / C}$ and $p 53^{C / R}$ ESCs compared to $p 53^{+/+}$ or $p 53^{+/ C}$ cells, whereas loss of $p 53 \mathrm{mRNA}$ and parallel reduction in the mRNA level of the $p 53$ target gene $p 21$ were confirmed in mutant ESC lines (Fig. 4c). The ESC line produced by acquired gene targeting in wild-type ESCs ('acKO' ESCs) and a congenital KO ESC line derived from heterozygous intercrosses (named coKO) were analyzed to determine whether some compensatory effect occurred in the coKO line. A microarray analysis showed that the coKO line had less divergent expression compared to the acKO line (acKO vs. coKO: 494 vs. 11 genes upregulated and 214 vs. 50 genes downregulated, Fig. 4e). Venn diagrams showing the overlap in genes identified in the two KO ESC datasets identified only five upregulated and 30 downregulated genes (Fig. 4e, and see Supplementary Table S2). Many of the downregulated genes in the $p 53^{C / C}$ ESCs were direct targets of p53, such as Puma, p21, Ccng1, Plk2, Phlda3, and Ptprv (Fig. 4d), whereas no genes for pluripotency or stemness were identified.

Chimera generation was used to investigate whether microinjection with these coKO ESCs could rescue mutant chimera development. Male ESC lines were also examined because homozygous males showed normal development. However, microinjection of both female cell lines ( $n=4,2$ cell lines) and male coKO ESC cell lines $(n=7,3$ cell lines) produced chimeras in which embryogenesis failed, similar to the acKO chimeras (Fig. 4f and Supplementary Table S1). The fraction of full-sized embryos (15/68 embryos: $23.1 \pm 4.0 \%$, $\mathrm{n}=9,5$ cell lines) was similar to that of acKO chimeras $(30 / 189$ embryos: $20.0 \pm 5.6 \%, n=14,5$ cell lines). These results indicate that lethality in mutant chimeras is due to abnormality of $p 53^{-/-}$ESCs.

$p 53^{-1-}$ ESCs are resistant to apoptosis and differentiation. To investigate susceptibility to apoptosis, flow cytometry to detect surface exposure of Annexin V was performed in ESCs under routine culture conditions using YPAC medium [Y, Y-27632 (ROCK inhibitor); P, PD0325901 (MEK inhibitor); A, A-83-01 (TGF- $\beta$ inhibitor); C, CHIR99021 (GSK3 inhibitor) $]^{12}$. A control treatment with G418 caused an increase in Annexin V-positive apoptotic cells whereas each of the $p 53$ genotype ESCs exhibited small population of the apoptotic cells $\left(p 53^{+/+}, 11.0 \pm 0.25 \%\right.$; $p 53^{+/ C}, 14.0 \pm 0.30 \%$; $553^{C / C}, 11.0 \pm 0.49 \%$ ) (Fig. 5a). Assays for colony formation and embryoid body (EB) formation were performed under differentiation culture conditions to examine the behavior of the mutant ESCs. There was no genotypedependent difference in the numbers of undifferentiated or differentiated colonies under conditions using YPAC medium and MEFs (Fig. 5b). Under culture conditions using Y media and MEFs (inhibitors $\mathrm{P}, \mathrm{A}$ and $\mathrm{C}$ were absent, Fig. $5 \mathrm{c}$ ), almost no $p 53^{+/+}$ undifferentiated colonies formed (1.7 \pm 0.9 colonies) but some $p 53^{+/ C}$ colonies were observed $(13.0 \pm 2.1$ colonies, $P<0.01)$. Few differentiated colonies of either genotype were formed. In contrast, $p 53^{\mathrm{C} / \mathrm{C}}$ cells formed a large number of both undifferentiated $(56.0 \pm$ 2.6 colonies, $P<0.0001$ vs. $p 53^{+/+} ; P<0.001$ vs. $\left.p 53^{+/ C}\right)$ and differentiated $\left(26.3 \pm 1.5\right.$ colonies, $P<0.0001$ vs. $p 53^{+/+} ; P<0.0001$ vs. $p 53^{+/ C}$ ) colonies (Fig. $5 \mathrm{c}$ ). These results suggest that $p 53^{-/-}$ESCs strongly maintain both undifferentiated state and self-renewal capacities while the differentiated cells are protected from apoptosis, consistent with the results shown in Figure 3a. Next, colony formation was assessed under culture conditions in which ESCs weakly attach to un-coated culture dishes in the absence of MEFs. Although undifferentiated colony formation was rare in both $p 53^{+/+}(11.3 \pm 3.5)$ and $p 53^{+/ C}(2.0 \pm 1.2)$ ESCs, a large number of $p 53^{C / C}$ ESCs formed colonies $(96.7 \pm 2.8$ colonies, $P<0.0001$ vs. $p 53^{+/+} ; P<0.0001$ vs. $p 53^{+/ C}$; Fig. 4 d). In the un-coated dishes in the absence of MEFs, differentiated colonies were rarely formed from any ESC genotype. Thus, the $p 53^{-1-}$ ESCs might have an increased capacity to adhere tightly to the culture dish and/or proliferate without the support of feeder cells.

When EB formation was examined, $p 53^{+/+}$EBs formed by day 3 underwent apoptosis over time in culture, resulting in few EBs remaining by day 7 relative to day 3 . In addition, Venus fluorescence was completely lost in these cells (Fig. 5e, left). In contrast, $p 53^{\mathrm{C} / C} \mathrm{EBs}$ were large in size and number, and maintained Venus fluorescence (Fig. 5e, right). The number of cells in $p 53^{C / C}$ EBs $\left(3.25 \times 10^{5}\right)$ was significantly larger than cell number in $p 53^{+/+}$EBs $\left(1.00 \times 10^{3}\right.$, $P<0.01)$ or $p 53^{+/ C}$ EBs $\left(2.47 \times 10^{4}, P<0.01\right)$. Moreover, these data indicate that $p 53^{C / C} \mathrm{EBs}$ actively proliferated because the cell number increased from $2.5 \times 10^{5}$ at day 0 to $3.25 \times 10^{5}$ by day 7 (Fig. $5 f$ ). This result suggests that $p 53^{\mathrm{C} / C}$ cells are able to proliferate even in the absence of cell attachment. $p 53^{+/ C}$ EBs showed an intermediate phenotype with significant differences from the other genotypes $\left(P<0.01\right.$ vs. $p 53^{+/+}, P<0.01$ vs. $\left.p 53^{C / C}\right)$.

Chromosomal instability in $p 53^{-/-}$cells. Next, karyotype analysis was performed in $p 53^{-/-}$cells. Although $p 53^{+/ C}$ ESCs maintained a normal karyotype $42, \mathrm{XX},[20]$, one $p 53^{\mathrm{C} / \mathrm{R} 2}$ ESC clone exhibited abnormal karyotype 42,XX, add(15)(q22)[20] (Fig. 6a, red square and arrow). Moreover, once the $p 53^{C / R 2}$ ESC clone differentiated under EB forming culture conditions for two weeks (Fig. 6b), an additional chromosomal aberration, 41,X,-X, add(15)(q22)[20], was found in all cells analyzed (Fig. 6a, blue square). In a $p 53^{\mathrm{C} / \mathrm{Cl}} \mathrm{ESC}$ clone, ESCs did not have an abnormal karyotype (42,XX[20]). However, cells derived from the $p 53^{C / C 1(E S C)}$ chimera in E14.0 rats displayed various chromosomal aberrations, such as $42, \mathrm{XX}$, add(1) (q52) [1], 42,XX, add(3)(p12) [1], 43,XX, + 16[1] or 42,X,-X, + mar[1]. In cell cultures, $p 53^{+/+}$cells derived from a recipient blastocyst were eliminated, resulting in occupation by $p 53^{C / C 1}$ cells with AmCyan 1 expression (Fig. 6c).

These findings demonstrate that $p 53^{-/-}$ESCs exhibit several features of abnormalities, such as blockage of differentiation, induction of chromosomal instability, and escape from apoptosis, which are facilitated when the cells differentiate. Thus, p53 is indispensable for embryonic development in the mutant chimeric models (Fig. 1f) but dispensable in the homozygous models due to bypassing an ESC state (Fig. 1e).

\section{Discussion}

Here, two distinct strategies were used to generate $p 53 \mathrm{KO}$ rats: conventionally generated homozygous $\mathrm{KO}$ and ESC-based mutant chimeras. In the homozyogous KO rats, NTDs such as exencephaly and spina bifida were observed. This is the first NTD model created in genetically modified rats. Previously, a 50\% reduction in the number of females relative to males at weaning was observed in $p 53$ homozygous $\mathrm{KO}$ mice $^{17}$. In contrast, in the present study, a $96 \%$ reduction in the number of $p 53$ homozygous $\mathrm{KO}$ female rats surviving to weaning relative to homozygous $\mathrm{KO}$ males was observed (Table 1). In rats, exencephaly occurred in a large fraction of the homozygous KO females (55\%; Table 1), whereas only $8-16 \%$ of homozygous KO female mouse embryos exhibited exencephaly ${ }^{17,18}$. The survival ratio and spina bifida phenotype observed in these exencephalic rat embryos suggests that this species exhibits more severe phenotypes than mice. We hypothesize that rats are more sensitive to the stress of DNA damage than mice. Consistent with this observation, rat ESCs are more sensitive to differentiation signals than mouse ESCs, which is one reason why rat ESCs were not established until 2008. Mouse ESCs are very stable compared to other species. In mouse, successful chimera contribution and germline transmission using $p 53$-null mouse iPSCs has been reported ${ }^{24,25}$. These results were unexpected, considering the vast amount of data regarding the effects of p53 on cell cycle arrest, apoptosis, and DNA 

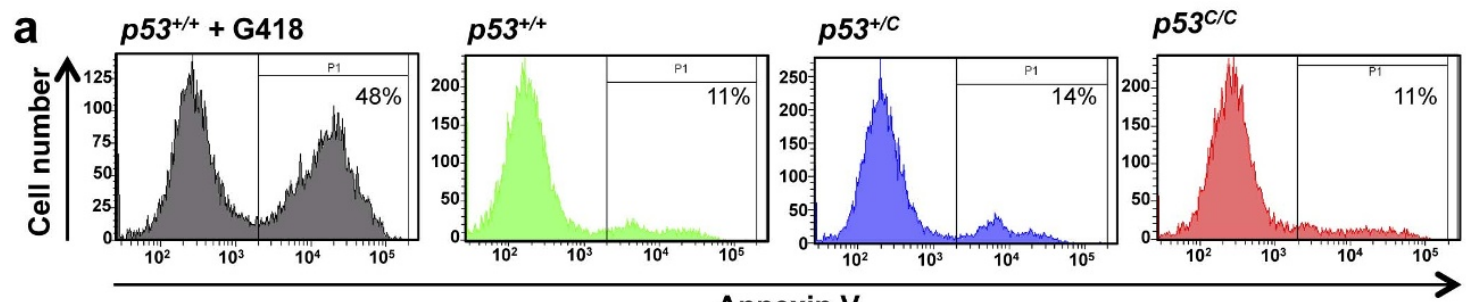

Annexin V
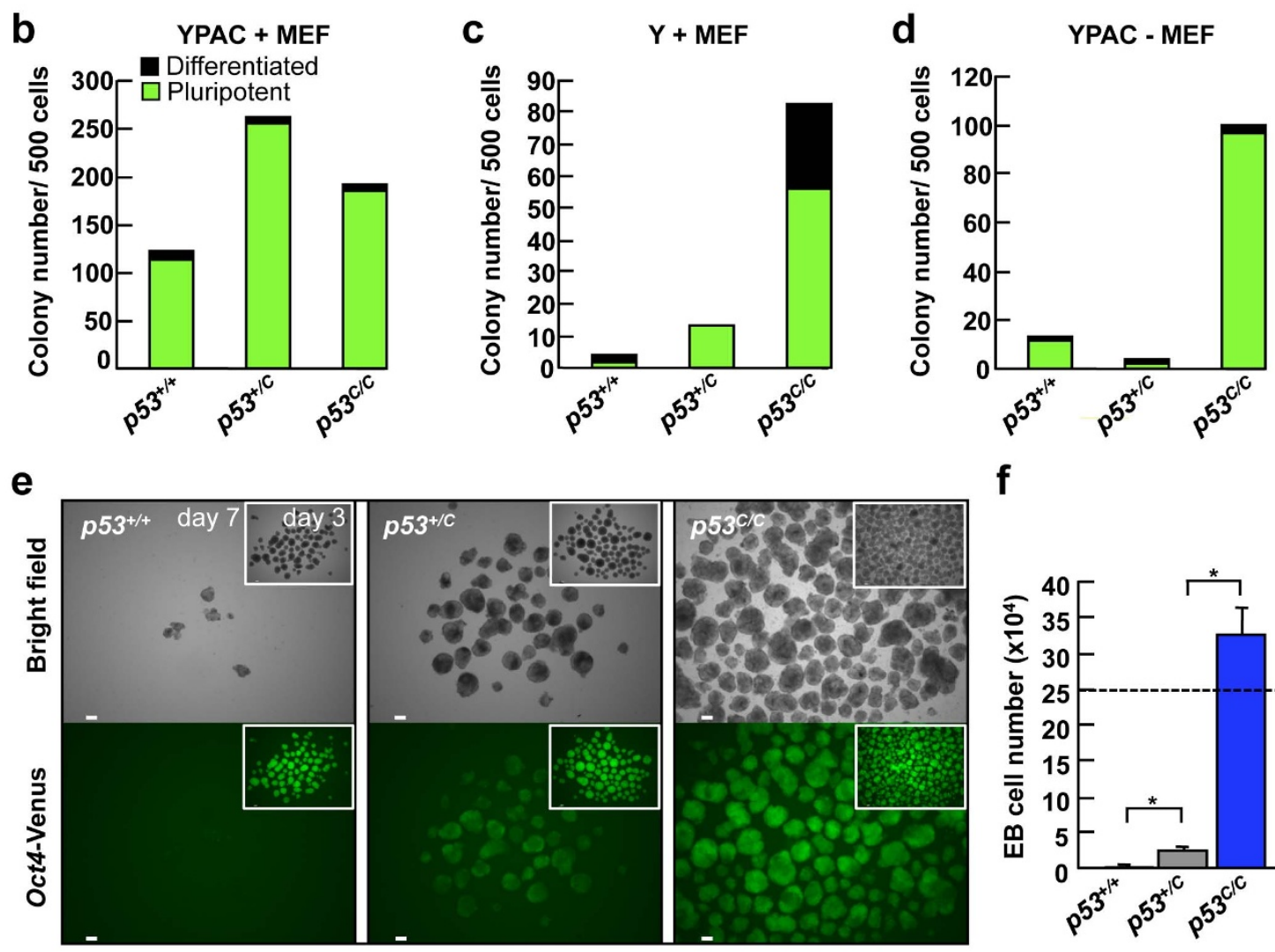

$f$

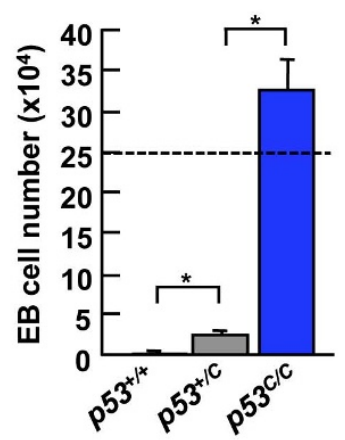

Figure 5 Colony and EB formation assays under differentiating conditions. (a) Flow cytometry analysis. Annexin V-Cy5 was used to detect apoptotic cells $(n=3)$. ( $b-d)$ Colony formation assay. Five hundred cells were cultured under normal conditions (YPAC+MEF; b), differentiation conditions $(\mathrm{Y}+\mathrm{MEF} ; \mathrm{c})$, or apoptosis-inducing conditions (YPAC-MEF; d). A green or black bar indicates pluripotent or differentiated colony number, respectively ( $\mathrm{n}=3$ or 4$)$. ( $\mathrm{e}, \mathrm{f})$ EB formation assay. EBs were formed from $2.5 \times 10^{5}$ cells (f, dotted line). Seven days after incubation without inhibitors, the cell number was counted (f, $\mathrm{n}=3$ ). Insets (e) indicate EBs at day 3. ${ }^{*}, P<0.01$. All scale bars $=100 \mu \mathrm{m}$.

repair. In contrast, in the present study, mutant chimeras generated with rat ESCs demonstrated a clear phenotype of embryonic lethality, consistent with the data presented here showing the downregulation of $p 53$ target genes, inhibition of apoptosis and differentiation, and increase in chromosomal instability in p53-null rat ESCs or ESC-derived cells.

The rat is considered to be a better model than the mouse for many complex disorders that are common in humans ${ }^{36}$ and is currently the primary animal model in many preclinical tests, especially those related to cardiovascular disease, diabetes, breast cancer, chronic inflammatory diseases, and age-related diseases ${ }^{20}$. Genetically modified rats are valuable platforms for the study of human physiology and disease. For example, in comparison to transgenic mice, transgenic rat models of Huntington disease not only present a more typical adult patient pathology but are also more suitable for in vivo metabolic and structural imaging ${ }^{20,37}$. In addition, $A p c$ knockout mice develop tumors primarily in the small intestine, whereas both humans and rats develop colon cancer as a result of the Apc mutation $^{38}$. These observations support the inconsistent phenotype of $p 53$ mutant chimeras between rats and mice, as shown in the present work, and suggest the importance of generating genetically modified rats to find novel gene functions.

In this study, the differences in the phenotypes of the p53 homozygous and mutant chimeric rat models were striking. Secondary mutations are accumulated in the mutant ESCs under in vitro culture conditions and in the differentiating cells during embryogenesis (Fig. 6). These aberrant cells are resistant to apoptosis due to p53 deficiency, which might lead to lethality of the mutant chimeras. These observations reflect the fact that a major p53 function is to be the "guardian of the genome". Thus, the mutant chimeric strategy may prove useful in identifying authentic and/or novel gene functions. Finally, the present study demonstrated that mutant chimeric models can be generated within one month, circumventing both the risks associated with producing successful germline transmission as well as the time frame required for breeding both chimeras and heterozygous animals. In the mutant chimeric method, double or triple gene knockouts can be generated in a few months. These new combination strategies using embryonic stem cells, the mutant 
a

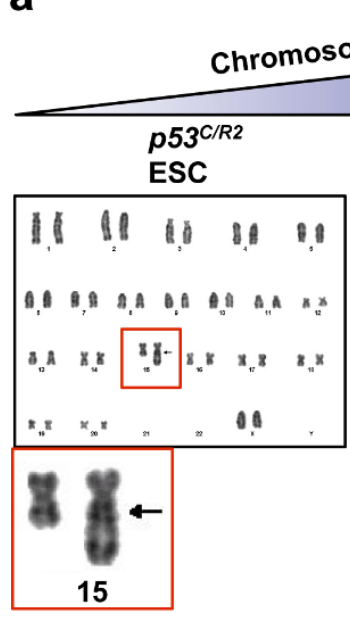

b

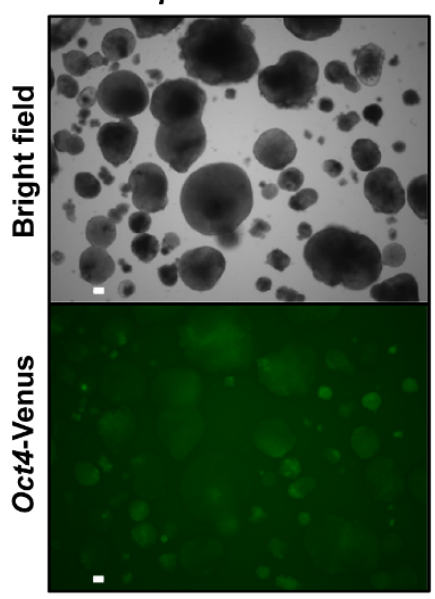

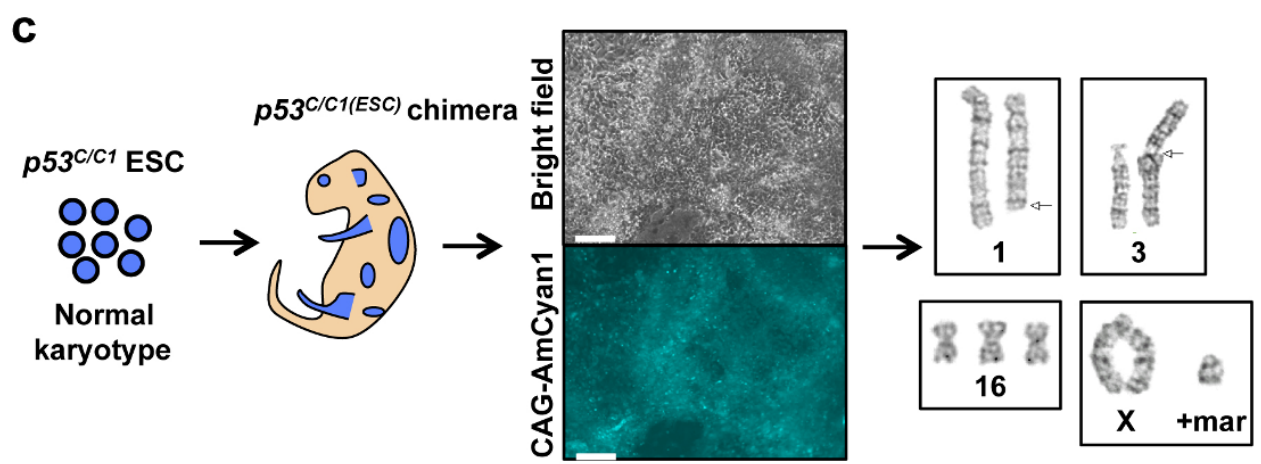

Figure $6 \mid$ Chromosomal instability in p53-null cells. (a) Cytogenetic analysis by G-band staining in $p 53^{C / R 2}$ ESCs and EB-derived differentiated $p 53^{C / R 2}$ cells. Abnormal chromosomes were indicated by red and blue squares. (b) Differentiated $p 53^{C / R 2}$ EBs lacking Venus fluorescence. (c) Aberrant chromosomes were observed in cells derived from a $p 53^{C / C 1(E S C)}$ chimera at E14.0.

chimeric method, and rats instead of mice will provide great insight into the novel functions of a large number of genes. The first example, shown here by deleting the $p 53$ gene, provided new, substantial evidence demonstrating that p53 functions not only as the "guardian of the genome", but also as the "guardian of the embryogenesis".

\section{Methods}

Media, feeder cells, animals, and primers. The YPAC medium was prepared by the addition of the following inhibitors: $10 \mu \mathrm{M}$ Y-27632 (WAKO), $1 \mu \mathrm{M}$ PD0325901 (Axon Medchem), $0.5 \mu \mathrm{M}$ A-83-01 (TOCRIS), and $3 \mu \mathrm{M}$ CHIR99021 (Axon Medchem) to a basic medium. The basic medium was composed of DMEM (including $110 \mathrm{mg} / \mathrm{L}$ sodium pyruvate and $200 \mathrm{mM}$ GlutaMAX, GIBCO), 20\% FBS (ES Cell Qualified Fetal Bovine Serum, Lot No. 1204059, GIBCO), 0.1 mM 2mercaptoethanol (SIGMA), $1 \%$ nonessential amino acid stock (GIBCO), and $1 \times$ antibiotic antimycotic (GIBCO). Mitomycin C-treated MEFs resistant to neomycin (Millipore) were used as feeders and maintained in 10\% FBS DMEM (Lot No. SFB301502, EQUITECH-BIO, INC.) with $1 \times$ antibiotic antimycotic. Animal experiments were performed in compliance with the guidelines of the Institute for Laboratory Animal Research, National Cancer Center Research Institute. These studies were approved by National Cancer Center Research Institute. All primer sequences are listed in Supplementary Table 3.

Generation of Oct4-Venus Tg rats and ESCs. Oct4-Venus Tg rats of the Wistar strain were generated via germline transmission of an Oct4-Venus ESC clone in the same manner described previously ${ }^{12}$. The Oct4-Venus ESC line derived from the Long-Evans Agouti (LEA) strain was generated in an earlier study ${ }^{12}$.

Establishment of rat ES cells from blastocysts. Rat blastocysts were gently flushed out from the uteri of E4.5 or E5.0 pregnant rats with a basic ES medium. After removal of the zona with acid Tyrode's solution (Ark Resource Co., Ltd.), whole blastocysts were plated onto 6-well plates and cultured on MEFs in basic ES medium with or without YPAC. After approximately 7 days, the blastocyst outgrowths were cut into pieces and replated under the same YPAC conditions. Emerging ESC colonies were then dissociated with Accutase (Innovative Cell Technologies, Inc.) and expanded. Domed and floated ESC colonies were detached from MEFs by pipetting, followed by routinely passaging every 3-4 days under MEF-YPAC conditions.

ZFN constructs and targeting plasmids. Custom-designed ZFN plasmids and ZFNencoding mRNA for the rat $p 53$ gene were purchased from Sigma-Aldrich. The design, cloning, and validation of the ZFNs were performed by Sigma-Aldrich. The ZFN pair recognizing exon 4 of the $p 53$ gene was $5^{\prime}$ : TTCTCCAGTCTTCCTCCAG, 3': ATTCTGGTAAGGAGCCGG. The targeting donor was composed of the CAGAmCyan1-IRES-Neo-pA or CAG-tdTomato-IRES-Neo-pA cassette with short homology 5' (736 bp) and 3' (711 bp) arms. These homology arms were amplified from rat genomic DNA using the KOD Ver.2 DNA polymerase PCR system (Toyobo). The sequences of these primers are listed in Supplementary Table S3. Both arms were set at several base pairs from a ZFN-induced cleavage site.

Introduction of the targeting vector and ZFNs into LEA rat ESCs and generation of $p 53$ heterozygous rats. To disrupt exon 4 of the $p 53$ gene, $5 \mu \mathrm{g}$ of ZFN-encoding mRNAs and $10 \mu \mathrm{g}$ of a targeting plasmid (5'arm-CAG-AmCyan1-IRES-Neo-pA$3^{\prime}$ arm cassette) were co-transfected into $6.5 \times 10^{5}$ of Oct4-Venus ESCs derived from a LEA female strain at passage 5 with a Mouse ESC Nucleofector Kit (Amaxa Inc.). The cells were plated on MEFs in YPAC medium with $2 \%$ Matrigel (BD Biosciences) to keep the ESC colonies adhered to the MEFs. One day after nucleofection, geneticin was added to the YPAC medium at $0.2 \mu \mathrm{g} / \mathrm{ml}$. Eleven days after nucleofection, geneticin-resistant colonies were selected using handmade capillaries and expanded. Picking and expanding seven colonies provided four $(57 \%)$ heterozygous $\left(p 53^{+/ C}\right)$ clones and two (29\%) homozygous clones harboring both knock-in and frame shift mutation alleles $\left(p 53^{C / Z}\right.$ ) (Supplementary Fig. S1a). The ZFN-induced frame shift mutation was identified by band shift in PCR analysis (Supplementary Fig. S1a, No. 6 clone, asterisk) and sequence analysis (Supplementary Fig. S1c) or using a SURVEYOR Mutation Detection Kit (Supplementary Fig. S1b, No. 8 clone). A $p 53^{+/ C}$ ESC clone was used for microinjection, resulting in the generation of $p 53^{+/ C}$ rats through chimeric germline transmission.

Introduction of the targeting vector and ZFNs into Wistar rat ESCs. For nucleofection, $10 \mu \mathrm{g}$ of a targeting plasmid (5' arm-CAG-AmCyan1-IRES-Neo-pA-3' arm 
cassette) and $5 \mu \mathrm{g}$ of ZFN-encoding mRNAs were co-transfected into $4.5 \times 10^{6}$ of Oct4-Venus ESCs (Wistar) at passage 3. As a control experiment, $10 \mu \mathrm{g}$ of the targeting plasmid without ZFNs was transfected into $4.5 \times 10^{6}$ of Oct4-Venus ESCs at passage 3. One out of $46(2.2 \%)$ clones was $p 53^{C / C}$, while seven of $46(15 \%)$ clones were $p 53^{C / Z}$. A sequence data revealed an 8 -bp deletion in the $p 53^{C / Z 1}$ clone (Figure S2B). This ZFN-induced small deletion was also confirmed by a downward band shift (indicated by asterisks in Supplementary Fig. S2a). As a control experiment, the targeting vector alone was introduced without ZFN. Although 14 geneticin-resistant colonies appeared, they did not achieve homologous recombination (Supplementary Fig. S2a, lane 5). Knockout clones were also produced using a 2nd-step recombination by introducing the $10 \mu \mathrm{g}$ of targeting plasmid ( $5^{\prime}$ arm-CAG-tdTomato-IRES-Neo$\mathrm{pA}-3^{\prime}$ arm cassette) and $5 \mu \mathrm{g}$ of ZFN-encoding mRNAs into $2.5 \times 10^{6}$ cells of a $p 53^{+/ C 3}$ ESC clone at passage 9 (Fig. 6). Eight red fluorescence (tdTomato)-positive clones were chosen without geneticin selection, and successful homologous recombination was achieved in three of these $\left(38 \%, p 53^{C / R}\right.$; Supplementary Fig. S2c).

Surveyor nuclease (Cel-I) assay. A ZFN target locus was amplified by PCR ( 35 cycles: $10 \mathrm{~s}$ denaturing at $98^{\circ} \mathrm{C}, 30 \mathrm{~s}$ annealing at $62^{\circ} \mathrm{C}$ and $1 \mathrm{~min}$ elongation at $72^{\circ} \mathrm{C}$ ) using primers 1 and 5 (Fig. 6 and Supplementary Table S3). The Cel-I assay was carried out following the manufacturer's protocol (TRANSGENOMIC, Inc.).

\section{ALP staining, immunohistochemistry (IHC) and Annexin V-apoptosis assay.} Cells were fixed in 4\% paraformaldehyde. ALP staining was performed with the Vector Blue substrate (Vector Labs) according to the manufacturer's instructions. Formalin-fixed and paraffin-embedded slides were stained with hematoxylin and eosin or used for IHC. Antigen retrieval was performed by autoclave in a sodium citrate buffer. The slides were incubated with Sox 2 (BioLegend, $1: 200$ ) or Cleaved Caspase-3 (Cell Signaling, 1/1000) primary antibody at $4^{\circ} \mathrm{C}$ overnight. The next day, after washing, the samples were incubated with horseradish peroxidase-conjugated secondary antibody for $1 \mathrm{~h}$. They were then washed and incubated with 3,3' diaminobenzidine tetrahydrochloride DAB (Thermo Scientific). An assay for apoptotic ESCs was performed using Annexin V-Cy5 following the manufacturer's protocol (BioVision). Pluripotent ESC colonies were solely harvested and dissociated with Accutase, followed by incubating 1-5 $\times 10^{5}$ cells with the Annexin V-Cy5 for $5 \mathrm{~min}$ in the dark.

Q-PCR analysis. Total RNA was isolated using ISOGEN (Nippongene). cDNA was synthesized with $2 \mu \mathrm{g}$ of the total RNA using Super Script III RT (Invitrogen) and oligo-dT primer (Invitrogen). cDNAs were used for PCR using Platinum SYBR Green qPCR SuperMix UDG (Invitrogen). Optimization of the q-PCR reaction was performed according to the manufacturer's instructions (PE Applied Biosystems, Tokyo, Japan). All quantitations were normalized to an endogenous control GAPDH.

Microarray analysis. A one-color microarray-based gene expression analysis system (Agilent Technologies) using SurePrint G3 Rat GE $8 \times 60$ K Kit containing 30507 probes (26930 genes) was used following the manufacturer's instructions.

EB formation. After ES cells were dissociated into single cells using Accutase, $5 \times 10^{5}$ cells were cultured in PAC medium. After overnight incubation, the EB contained media were separated and cultured in media with or without PAC on a low cellbinding dish (NUNC). After 7 days of incubation, the cell number of EBs was counted after dissociation with Accutase.

Chimera production. In all blastocyst injection experiments, 12 ESCs were injected into E4.5 blastocysts. YPAC or PAC inhibitors were constantly included in media during both microinjection and blastocyst incubation. ESC-injected blastocysts were transferred to E3.5 pseudo-pregnant rats. The contribution of ESCs to the resulting chimeras was determined by the appearance of coat-color or fluorescence.

Karyotype analyses in $p 53^{-/-}$cells. G-band staining was performed in cultured cells from embryos, ESCs, or ESC-derived differentiated cells. Head of E14.0 chimeric embryo was dissociated with Accutase and karyotype analysis was examined in the cells at passage 4. $p 53^{C / R 2}$ ESCs at five passages after the generation of the genetargeted null mutation or EB-derived differentiated $p 53^{C / R 2}$ cells at seven passages were analyzed. EBs were formed at passage 5 and cultured for 2 weeks, followed by two passages to expand the cells. The differentiated state was confirmed by a loss of Oct4-Venus expression, as well as by the cell morphology.

Statistical analysis. Results are given as the mean \pm SD. Statistical analysis was conducted using Student $t$-tests. $P<0.05$ was considered significant.

1. Cozzi, J. et al. Pronuclear DNA injection for the production of transgenic rats. Methods Mol. Biol. 561, 73-88 (2009).

2. Dann, C. T., Alvarado, A. L., Hammer, R. E. \& Garbers, D. L. Heritable and stable gene knockdown in rats. Proc. Natl. Acad. Sci. U S A. 103, 11246-11251 (2006).

3. Zan, Y. et al. Production of knockout rats using ENU mutagenesis and a yeastbased screening assay. Nat. Biotechnol. 21, 645-651 (2003).

4. van Boxtel, R., Gould, M. N., Cuppen, E. \& Smits, B. M. ENU mutagenesis to generate genetically modified rat models. Methods Mol. Biol. 597, 151-167 (2010).

5. Kitada, K. et al. Transposon-tagged mutagenesis in the rat. Nat. Methods $\mathbf{4}$, 131-133 (2007).
6. Kitada, K., Keng, V. W., Takeda, J. \& Horie, K. Generating mutant rats using the Sleeping Beauty transposon system. Methods 49, 236-242 (2009).

7. Izsvák, Z. et al. Generating knockout rats by transposon mutagenesis in spermatogonial stem cells. Nat. Methods 7, 443-445 (2010).

8. Geurts, A. M. et al. Knockout rats via embryo microinjection of zinc-finger nucleases. Science 325, 433 (2009).

9. Mashimo, T. et al. Generation of knockout rats with X-linked severe combined immunodeficiency (X-SCID) using zinc-finger nucleases. PLoS One 5, e8870 (2010).

10. Buehr, M. et al. Capture of authentic embryonic stem cells from rat blastocysts. Cell 135, 1287-1298 (2008).

11. Li, P. et al. Germline competent embryonic stem cells derived from rat blastocysts. Cell 135, 1299-1310 (2008).

12. Kawamata, M. \& Ochiya, T. Generation of genetically modified rats from embryonic stem cells. Proc. Natl. Acad. Sci. USA 107, 14223-14228 (2010).

13. Hamanaka, S. et al. Generation of germline-competent rat induced pluripotent stem cells. PLoS One 6, e22008 (2011).

14. Hirabayashi, M. et al. Rat transgenesis via embryonic stem cells electroporated with the Kusabira-orange gene. Mol. Reprod. Dev. 77, 474 (2010).

15. Tong, C., Li, P., Wu, N. L., Yan, Y. \& Ying, Q. L. Production of p53 gene knockout rats by homologous recombination in embryonic stem cells. Nature 467, 211-213 (2010).

16. Donehower, L. A. et al. Mice deficient for p53 are developmentally normal but susceptible to spontaneous tumours. Nature 356, 215-221 (1992).

17. Armstrong, J. F., Kaufman, M. H., Harrison, D. J. \& Clarke, A. R. High-frequency developmental abnormalities in p53-deficient mice. Curr. Biol. 5, 931-936 (1995).

18. Sah, V.P. et al. A subset of p53-deficient embryos exhibit exencephaly. Nat. Genet 10, 175-180 (1995).

19. Donehower, L. A. \& Lozano, G. 20 years studying p53 functions in genetically engineered mice. Nat. Rev. Cancer 9, 831-841 (2009).

20. Huang, G. et al. Beyond knockout rats: new insights into finer genome manipulation in rats. Cell Cycle 10, 1059-1066 (2011).

21. van Boxtel, R. et al. Homozygous and heterozygous p53 knockout rats develop metastasizing sarcomas with high frequency. Am. J. Pathol. 179, 1616-1622 (2011).

22. Puzio-Kuter, A. M. \& Levine, A. J. Stem cell biology meets p53. Nat. Biotechnol. 27, 914-915 (2009)

23. Lin, T. et al. p53 induces differentiation of mouse embryonic stem cells by suppressing Nanog expression. Nat. Cell Biol. 7, 165-171 (2005).

24. Hong et al. Suppression of induced pluripotent stem cell generation by the p53-p21 pathway. Nature 460, 1132-1135 (2009).

25. Marion et al. A p53-mediated DNA damage response limits reprogramming to ensure iPS cell genomic integrity. Nature 460, 1149-1153 (2009).

26. Kawamata, M. \& Ochiya, T. Establishment of embryonic stem cells from rat blastocysts. Methods Mol. Biol. 597, 169-177 (2010).

27. Kawamata, M. \& Ochiya, T. Gene-manipulated embryonic stem cells for rat transgenesis. Cell Mol. Life Sci. 68, 1911-1915 (2011).

28. Hosako, H. et al. The roles of p53 and p21 in normal development and hyperthermia-induced malformations. Birth Defects Res. B Dev. Reprod. Toxicol. 86, 40-47 (2009)

29. Chen, X. et al. Sex difference in neural tube defects in p53-null mice is caused by differences in the complement of X not Y genes. Dev. Neurobiol. 68, 265-273 (2008).

30. Takemoto, T. et al. Tbx6-dependent Sox2 regulation determines neural or mesodermal fate in axial stem cells. Nature 470, 394-398 (2011).

31. Miyagi, S. et al. The Sox 2 regulatory region 2 functions as a neural stem cellspecific enhancer in the telencephalon. J. Biol. Chem. 281, 13374-13381 (2006).

32. Hockemeyer, D. et al. Efficient targeting of expressed and silent genes in human ESCs and iPSCs using zinc-finger nucleases. Nat. Biotechnol. 27, 851-857 (2009).

33. Soldner, F. et al. Generation of Isogenic Pluripotent Stem Cells Differing Exclusively at Two Early Onset Parkinson Point Mutations. Cell 146, 318-331 (2011).

34. Meyer, M., de Angelis, M. H., Wurst, W. \& Kühn, R. Gene targeting by homologous recombination in mouse zygotes mediated by zinc-finger nucleases. Proc. Natl. Acad. Sci. USA 107, 15022-15026 (2010).

35. Cui, X. et al. Targeted integration in rat and mouse embryos with zinc-finger nucleases. Nat. Biotechnol. 29, 64-67 (2011).

36. Abbott, A. Return to the rat. Nature 460, 788 (2009)

37. von Horsten, S. et al. Transgenic rat model of Huntington's disease. Hum. Mol. Genet. 12, 617-24 (2003).

38. Amos-Landgraf, J. M. et al. A target-selected Apc-mutant rat kindred enhances the modeling of familial human colon cancer. Proc. Natl. Acad. Sci. U.S.A. 104 4036-41 (2007)

\section{Acknowledgements}

We thank F. Takeshita, T. Katsuda, K. Hagiwara, Y. Yoshioka, R. Takahashi, N. Kosaka, H Tsuda, H. Sasaki and Y. Tamai for their technical advice. This work is supported by a Grant-in-Aid from the Third-Term Comprehensive 10-Year Strategy for Cancer Control. 


\section{Author contributions}

M.K. designed and performed experiments. M.K. and T.O. wrote the manuscript. T.O. supervised the project.

\section{Additional information}

Supplementary information accompanies this paper at http://www.nature.com/ scientificreports
Competing financial interests: The authors declare no competing financial interests.

License: This work is licensed under a Creative Commons

Attribution-NonCommercial-ShareALike 3.0 Unported License. To view a copy of this license, visit http://creativecommons.org/licenses/by-nc-sa/3.0/

How to cite this article: Kawamata, M. \& Ochiya, T. Two distinct knockout approaches highlight a critical role for p53 in rat development. Sci. Rep. 2, 945; DOI:10.1038/srep00945 (2012). 